\title{
AN IDEMPOTENT COMPLETION FUNCTOR IN HOMOTOPY THEORY
}

BY

\author{
HAROLD M. HASTINGS ${ }^{1}$
}

\begin{abstract}
We observe that Artin-Mazur style $R$-completions ( $R$ is a commutative ring with identity) induce analogous idempotent completions on the weak prohomotopy category pro-Ho(Top). Because Ho(Top) is a subcategory of pro-Ho(Top) and pro-Ho(Top) is closely related to the topologized homotopy category of J. F. Adams and D. Sullivan, our construction represents the Sullivan completions as homotopy limits of idempotent functors. In addition, we show that the Sullivan completion is idempotent on those spaces (in analogy with the Bousfield and $\mathrm{Kan} R_{\infty}$-completion on $R$-good spaces) for which its cohomology with coefficients in $R$ agrees with that of our Artin-Mazur style completion. Finally, we rigidify the Artin-Mazur completion to obtain an idempotent Artin-Mazur completion on a category of generalized prospaces which preserves fibration and suitably defined cofibration sequences. (Our previous results on idempotency and factorization lift to the rigid completion.) Our results answer questions of Adams, Sullivan, and, later, A. Deleanu.
\end{abstract}

1. Introduction. In $1967 \mathrm{M}$. Artin and B. Mazur [5] introduced a profinite completion into algebraic topology in order to study étale homotopy theory. This completion is a functor

$$
\hat{\text { AM }}: \mathrm{Ho}(\text { Top) } \rightarrow \text { pro-Ho(Top) }
$$

which associates to each space $X$ the category of all finite spaces under $X$. (Here Top denotes the category of pointed spaces and pro-Ho(Top) is the category of inverse systems over Ho(Top).) A space is called finite if it has the (pointed) homotopy type of a pointed $\mathrm{CW}$ complex with only finitely many nonzero homotopy groups, each finite. Later, D. Sullivan [23, 24] showed that the Artin-Mazur completion of a space $X$ induced a representable functor

$$
W \rightarrow \lim \left[W, X_{\mathrm{AM}}^{\wedge}\right],
$$

and took the representing object as the completion $X_{\text {Sul }}^{\wedge}$ of $X$. See also D. Quillen's [22] profinite and pro- $p$ completions. Sullivan used a topologized homotopy category (see also J. F. Adams [2, 3]), showed that the Artin-Mazur completion induced a compact Brownian functor (1.2), and proved a representability theorem for such functors. Essentially, the Sullivan completion is the homotopy limit (A. K. Bousfield and D. M. Kan [7, Chapter XI], D. A. Edwards and the author [12, Chapter 4]) of the Artin-Mazur completion; cf. [13].

Received by the editors November 1, 1983.

1980 Mathematics Subject Classification. Primary 55P60; Secondary 14F30, 55N05.

${ }^{1}$ Partially supported by NSF Grant MCS8102053. 
Later, Bousfield and Kan [7, Chapter I] introduced a different, rigid completion, the $R_{S}$-tower $\left\{R_{S} X\right\}$ of a space $X$, and observed that $\left\{R_{S} X\right\}$ is cofinal in an Artin-Mazur type $R$-completion. Here $R$ is a commutative ring with identity. We call $\left\{R_{S} X\right\}$ rigid because the construction of $\left\{R_{S} X\right\}$ yields a functor into Ho(pro-Top) (see [12, Chapter 3] for Ho(pro-Top)). Bousfield and Kan also studied the homotopy limit $R_{\infty} X$ of $\left\{R_{S} X\right\}$; this is essentially a Sullivan completion for coefficients in $R$.

In the 1973 Tokyo conference on manifolds, Adams [1] asked several questions about the Sullivan completion, leading up to the possible existence of an idempotent completion functor on the topologized homotopy category. (In this category the morphisms from $X$ to $Y$ form a toplogical space of homotopy classes of maps, and for any morphism $W \rightarrow X$, the induced morphism $[X, Y] \rightarrow[W, Y]$ is continuous.) Later, developing the "genetics of homotopy theory", Sullivan [24] remarked that a simple rigid completion functor could prove useful. A. Deleanu [9] defined the Sullivan completion on the topologized homotopy category, but left the question of idempotency open.

The study of Sullivan completions in the toplogized homotopy category is closely related to their study in pro-Ho(Top). Given a map $f: X \rightarrow Y$ in the topologized category, and a map $g: Y \rightarrow F$, with $F$ a finite space, the " $g$ "-neighborhood of $f$ consists of all maps $h: X \rightarrow Y$ with $g f \simeq g h$. Since the Sullivan completion of a space $Y$ is defined by the finite spaces under $Y$, Sullivan completions in the topologized homotopy category and in the prohomotopy category convey the same information. It is, however, simpler to work in prohomotopy.

We first address the existence of an idempotent completion functor by observing the following in §2; see also Artin and Mazur [5, Theorem 3.4].

(1.3) Theorem A. The Artin-Mazur completion extends to an idempotent completion on the category pro-Ho(Top) of inverse systems over the homotopy category.

We also define similar, idempotent, Artin-Mazur style $p$-finite and $R$-completions (as above, $R$ is a commutative ring with identity), and prove adjointness (cf. Adams [1]). We also relate pro-objects to topologized objects in the homotopy category, largely following Sullivan, thus identifying the Sullivan limit as a homotopy limit (cf. [12, Chapter 4; 13]). Thus Theorem A answers Adams's questions about idempotent completions.

In considering the failure of the idempotency for their $R_{\infty}$-completion, Bousfield and Kan defined good and bad spaces. A space $X$ is called $R$-good [7, Chapter I] if the natural map

$$
R_{\infty} X \rightarrow R_{\infty} R_{\infty} X
$$

is a homotopy equivalence. Nilpotent spaces are $R$-good for all $R$; the projective plane and some finite wedge of circles are not $Z$-good [7, Chapter IV]. We shall show that the failure of all spaces to be good arises from taking homotopy limits of Artin-Mazur completions, or, equivalently, from forgetting the Adams-Sullivan topologies on these homotopy limits. More precisely, we slightly alter the BousfieldKan definition of $R$-good as follows. 
(1.5) Definition. A space $X$ is called Sullivan $R$-good if the natural map of Sullivan $R$-completions,

$$
X_{\text {Sul }}^{\wedge} \rightarrow X_{\text {Sul }}^{\wedge} \hat{\text { Sul }},
$$

is a homotopy equivalence, or, more loosely, if the Sullivan $R$-completion is idempotent on that space.

In $\$ 3$ we prove the following.

(1.6) Theorem B. A space $X$ is Sullivan R-good if and only if the natural map from the Sullivan R-completion to the Artin-Mazur R-completion induces isomorphisms on cohomology with coefficients in $R$.

Sullivan [24] had also observed that his completion preserved fibrations, but did not, in general preserve cofibrations. Unfortunately, the weak prohomotopy cateory pro-Ho(Top) does not admit suitable cofibre and fibre sequences (cf. D. Quillen [21]). We therefore need another prohomotopy category to study the effect of the Artin-Mazur completion on cofibrations and fibrations. Our strong prohomotopy category Ho(pro-Top), defined with D. A. Edwards [12, Chapter 3] using Quillen's theory of model categories, does admit these cofibre and fibre sequences. However, the Artin-Mazur completion does not induce a completion into Ho(pro-Top) because the required equalizers do not exist; see [15]. We therefore define a suitable intermediate category gpro-Top of generalized prospaces in $\S 4$. We then observe ( $\$ 5)$ that Theorem A still holds for gpro-Top and its homotopy category Ho(gpro-Top) by rigidifying the Artin-Mazur completion. Details were sketched in [15, §6]. We define the required cofibration and fibration sequences in $\$ 6$ and then prove the following in $\$ 7$.

(1.7) THEOREM C. The rigid Artin-Mazur completion preserves cofibration sequences.

(1.8) TheOREM D. The rigid Artin-Mazur completion preserves fibration sequences.

(1.9) Remarks. (i) Some care is needed in interpreting Theorem C. Although the fibre of a map between generalized Eilenberg-Mac Lane spaces is again such a space, this is not true of the cofibre. We therefore shall define a category of generalized prospaces over generalized Eilenberg-Mac Lane spaces, whose homotopy groups are finite graded $R$-modules, and take the required cofibres in this category.

(ii) Theorem $\mathrm{C}$ shows that the failure of Sullivan's completion to preserve cofibres lies in the failure of the homotopy limit to preserve cofibres.

We acknowledge helpful conversations with A. Heller.

2. The Artin-Mazur completion. We review the definition of the Artin-Mazur completion, and observe that it readily extends to an idempotent finite completion functor on pro-Ho(Top), as well as analogous $p$-finite and $R$-finite completion functors. We also relate completions into pro-Ho(Top) to completions as topologized objects. In combination with Sullivan's representability theorem for compact Brownian functors [24], this provides an idempotent factorization of the Sullivan completion. Our observation also suggests that the questions about rigidity and 
cofibrations and fibrations could be answered by suitably lifting the Artin-Mazur completion.

(2.1) Definition. A space is called finite if it has the homotopy type of a pointed, connected CW complex and its homotopy groups form a finite graded group. We shall write Fin for the full subcategory of Top generated by the finite spaces, and $\mathrm{Ho}$ (Fin) for the induced homotopy category. The category $p$-Fin of $p$-finite spaces is the full subcategory of finite spaces all of whose homotopy groups are (finite) $p$-groups. For any finite commutative ring with identity $R$ or subfield of $Q$, the category $R$-Fin of $R$-finite spaces is the full subcategory of those spaces whose homotopy groups form a finitely generated (graded) $R$-module. The homotopy categories of $p$-finite and $R$-finite spaces are defined analogously with Ho(Fin).

(2.2) REMARKS. To be technically correct, choose a fixed set of representatives for isomophism classes of finite (or $p$-finite or $R$-finite) spaces, and consider the small, full subcategory which they generate. Call these categories Fin, $p$-Fin, $R$-Fin, respectively.

We recall the original definition of the Artin-Mazur [5] completion in the language of comma categories (cf. S. Mac Lane [17]).

(2.3) Definition. The Artin-Mazur (finite) completion of a space $X$ is given by

$$
X_{\mathrm{AM}}^{\wedge}=\{X \downarrow \operatorname{Ho}(\text { Fin }) \rightarrow \operatorname{Ho}(\text { Fin })\} .
$$

There are similar $p$-finite and $R$-finite completions.

Artin and Mazur proved that the indexing category $X \downarrow$ Ho(Fin) is filtering. Hence, Definition (2.3) yields a functor from Ho(Top) to pro-Ho(Fin), a full subcategory of pro-Ho(Top). We shall define Artin-Mazur finite, $p$-finite, and $R$-finite completions on pro-Ho(Top) by the same formula (2.4), where Fin is replaced by the appropriate category of finite spaces, and continue to write $\hat{A M}$ to denote this extension of the usual Artin-Mazur completion. It is easy to show the following.

(2.5) LeMma. For any prospace $X$ in pro-Ho(Top), the comma category $X \downarrow$ Ho(Fin) is filtering, as are analogous comma categories over $p$-Fin and $R$-Fin.

Proof. Proceed as in the proof of the analogous result for the usual Artin-Mazur completion [5]. The required product cones (common predecessors) exist because Fin is closed under products. The required equalizer cones exist because Fin is closed, admits homotopy pullbacks, and hence admits homotopy equalizers.

(2.6) Proposition. The above construction extends to a functor

$$
\hat{\text { AM }}: \text { pro-Ho(Top) } \rightarrow \text { pro-Ho(Fin }) \subset \text { pro-Ho(Top) }
$$

and a natural transformation id $\rightarrow \hat{\mathrm{AM}}$. There are $p$-finite and $R$-finite completion functors with similar properties, denoted ${ }_{p}^{\wedge}$ and ${ }_{R}$, respectively.

The proof is easy and omitted.

(2.7) THEOREM. The completion functor $\hat{\mathrm{AM}}$ is idempotent; that is, both natural transformations $\hat{\mathrm{AM}} \rightarrow \hat{\mathrm{AM}}^{2}$ are natural isomorphisms, similarly for $\hat{p}_{\mathrm{p}}$ and $\hat{R}_{R}$. 
Proof. We consider the finite completion; the others are similar. By comma category formalism, there is a natural isomorphism of the indexing categories of $\hat{A M}$ and $\hat{A M}^{2}$ :

$$
\{(X \downarrow \text { Ho(Fin) }) \rightarrow \operatorname{Ho}(\text { Fin })\} \downarrow \text { Ho(Fin) } \rightarrow X \downarrow \text { Ho(Fin) }
$$

The conclusion follows.

The above results imply:

(2.8) TheOREm A. The Artin-Mazur completion extends to an idempotent completion on the category pro-Ho(Top).

(2.9) Proposition. The functor $\hat{\mathrm{AM}}$ : pro-Ho(Top) $\rightarrow$ pro-Ho(Fin) is full.

Proof. Let $X$ be a finite space. Then (the identity map of) $X$ is cofinal in $X \downarrow$ Ho(Fin). Consequently, for any profinite space $X$ ( $X \in$ obj pro-Ho(Fin)), (the identity map of) $X$, as an indexing category, is cofinal in $X \downarrow$ Ho(Fin). The conclusion follows.

(2.10) CoROllaRY. The functor $\hat{\text { AM }}$ : pro-Ho(Top) $\rightarrow$ pro-Ho(Fin) is a reflection (left adjoint to the inclusion).

Thus our "extended" Artin-Mazur completion satisfies Adams's criteria [1] for a good completion functor. Similar results hold for the $p$-finite and $R$-completions.

Although limits do not exist, in general, on pro-Ho(Top) (D. Puppe showed me an example, which I gave in [15]), objects in pro-Ho(Fin) do have limits in Ho(Top). This is Sullivan's theorem [24]; his proof involves a version of Brown's representability theorem for half-exact functors taking values in compact spaces. Furthermore, these "Sullivan" limits satisfy the usual adjointness relation where it is defined: for $X$ in Top, $Y$ in pro-Ho(Fin), and $J: H o(T o p) ~ \rightarrow$ pro-Ho(Top) the inclusion,

$$
\text { pro-Ho(Top) }(J X, Y)=\operatorname{Ho}(\mathrm{Top})\left(X, \lim _{\text {Sul }} Y\right) \text {. }
$$

We shall therefore write holim for the Sullivan limit, $\lim _{\text {Sul }}$, and use the formula

$$
\hat{\text { Sul }}=\operatorname{holim} \circ \hat{A M} \circ J
$$

to relate the Sullivan completion $\hat{\text { Sul }}$ to the extended Artin-Mazur completion.

For $Y$ in pro-Ho(Fin), the homotopy limit holim $Y$ is "topologized" in the Adams-Sullivan sense using the limit of the topologies on finite spaces $F$ which make $[K, F]$ discrete for any finite $\mathrm{CW}$ complex $K$. In this sense the prospace $X$ and its "topologized" Sullivan limit $\lim _{\text {Sul }} X$ carry the same information, in analogy with the Lie series of a topological group. We can also use $p$-finite or, for finite rings $R$, $R$-finite, spaces here. In this sense we have just answered Adams's questions (see also [9]) about an idempotent completion for topologized objects.

We cannot, however, address questions about cofibrations and fibrations within pro-Ho(Top). The nonexistence of these constructions with pro-Ho(Top) motivated D. A. Edwards and the author [12, Chapter 3] to develop a closed model structure on pro-Top, and to use this structure and Quillen's [21] theory of closed model 
categories to define the resulting homotopy category Ho(pro-Top). It would therefore be desirable to define a rigid Artin-Mazur completion by the formula

$$
X^{\wedge}=\{X \downarrow \text { Fin } \rightarrow \text { Fin }\} .
$$

However, the equalizer of two maps between finite spaces need not be finite, and this implies that the indexing category $X \downarrow$ Fin is not filtering. Therefore, formula (2.13) does not yield a functor into the strong prohomotopy category. We shall develop a prohomotopy category which avoids this difficulty in the next section.

(2.14) Remarks. The indexing category $X \downarrow$ Fin can be shown to be homotopy filtering, and thus formula (2.13) should yield a "coherent" Artin-Mazur completion into a suitable coherent homotopy category of inverse systems. See R. Vogt [25] for the use of coherency in homotopy limits and colimits, and T. Porter [20] and J. Lisica and S. Mardešić [16] for possible coherent target categories. However, this approach seems difficult and unnecessary.

3. Good spaces-Proof of Theorem B. Let $R$ be a commutative ring with identity. Recall that Bousfield and Kan [7, Chapter I] called a space $X R$-good if the natural map $X \rightarrow R_{\infty} X$ is an isomorphism, and called $X R$-bad otherwise. Recall that the Sullivan completion is the homotopy limit of our idempotent Artin-Mazur type completion, the Bousfield-Kan $R_{\infty}$-completion is the homotopy limit of their $\left\{R_{S}\right\}$ completion and the $\left\{R_{S}\right\}$-completion is cofinal in the Sullivan completion. It is therefore natural to conjecture that a space is $R$-good if and only if the Sullivan and Artin-Mazur completions are suitably equivalent. We shall prove such a result for Sullivan $R$-good spaces after first discussing when two prospaces have the same Artin-Mazur $R$-completion. We begin with two easy lemmas, whose proofs are omitted.

(3.1) LemMA. $H^{*}(; R)$ is represented by $K(R, n)$ on pro-Ho(Top).

(3.2) Lemma. Let $F \rightarrow E \rightarrow B$ be a fibre sequence in Top. Then the sequence $[X, F] \rightarrow[X, E] \rightarrow[X, B]$ is exact at $[X, E]$ for any $X$ in pro-Ho(Top).

(3.3) TheOREM. Let $f: X \rightarrow Y$ be a map in pro-Ho(Top). Then $f$ induces an equivalence on Artin-Mazur R-completions if and only if $f$ induces isomorphisms on cohomology with coefficients in $R$. Similar results hold for p-completions, and the finite completion where integral coefficients are used.

Proof. We consider the case of $R$-completions, and leave the modifications in the other cases to the reader. First suppose that $f$ induces isomorphisms on $H^{*}(; R)$. Then for any Eilenberg-Mac Lane space of the form $K(R, n), f$ induces an isomorphism

$$
[f, K(R, n)]:[Y, K(R, n)] \rightarrow[X, K(R, n)] .
$$

Now use induction, and the five-lemma applied to the diagrams of the form

$$
\begin{aligned}
& {[Y, \Omega B] \rightarrow[Y, K(R, n)] \rightarrow[Y, E] \rightarrow[Y, B] \rightarrow[Y, K(R, n+1)]}
\end{aligned}
$$

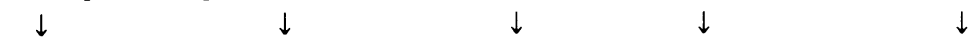

$$
\begin{aligned}
& {[X, \Omega B] \rightarrow[X, K(R, n)] \rightarrow[X, E] \rightarrow[X, B] \rightarrow[X, K(R, n+1)]}
\end{aligned}
$$


induced from fibration sequences $K(R, n) \rightarrow E \rightarrow B$, where $B$ and, hence, $E$ are $R$-finite spaces, to conclude that $f$ induces isomorphisms on homotopy classes of maps into $R$-finite spaces. (The group structure on Eilenberg-Mac Lane spaces and the $H$-space structure of loop spaces are used implicitly in the five-lemma above.) This implies that $f$ induces an equivalence of Artin-Mazur $R$-completions, as required.

For the converse, assume that $f$ induces isomorphisms on the Artin-Mazur $R$-completions. For any map $X \rightarrow K(R, n)$, representing a cohomology class, there is then a commutative diagram in pro-Ho(Top),

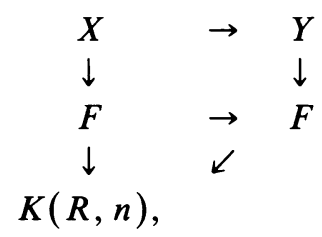

where $F$ is a finite $R$-space. This implies that $f$ induces an isomorphism on $H^{*}(; R)$, as required.

(3.4) Remarks. Bousfield and Kan [7, Chapter I] prove a similar result for their $R$-completion, except that cohomology is replaced by homology.

(3.5) THEOREM B. A space $X$ is Sullivan $R$-good if and only if the natural map

$$
\pi: X_{\text {Sul }, R}^{\wedge}=\operatorname{holim} X_{R}^{\wedge} \rightarrow X_{R}^{\wedge}
$$

induces isomorphisms on cohomology with coefficients in $R$.

Proof. First suppose that $\pi$ induces isomorphisms on $H^{*}(; R)$. By Theorem (3.3) the induced map

$$
X_{\text {Sul } \hat{R}}^{\wedge} \rightarrow X_{R}^{\wedge}{ }^{2} \cong X_{R}^{\wedge}
$$

is an isomorphism in pro-Ho(Top). Applying homotopy limits yields the required homotopy equivalence $X_{\text {Sul }}^{\wedge} \rightarrow X_{\text {Sul }}^{\wedge}$ (it is easy to see that this map is a homotopy inverse to the natural map $X_{\text {Sul }}^{\wedge} \rightarrow X_{\text {Sul }}^{\wedge}{ }^{2}$ ).

Conversely, suppose that $X$ is a Sullivan $R$-good space. Then the natural map $X_{\text {Sul }}^{\wedge} \rightarrow X_{\text {Sul }}^{\wedge}{ }^{2}$ is an isomorphism in pro-Ho(Top). We may rewrite this equivalence as

$$
\text { holim } \circ{ }_{R} \circ X \rightarrow \text { holim } \circ{ }_{R} \circ \text { holim } \circ{ }_{R} \circ X .
$$

We begin by stripping off the first holim, using the following lemma.

(3.7) LEMMA. The map (3.6) induces an equivalence in pro-Ho(Top):

$$
{ }_{R} \circ X \rightarrow{ }_{R} \circ \text { holim } \circ{ }_{R} \circ X \text {. }
$$

Proof. First consider the Bousfield-Kan spectral sequence [7, Chapter XI] of a prospace $Y$,

$$
E_{2}^{p, q}=\lim ^{p} \text { pro- } \pi_{q} Y,
$$

abutting to $\pi_{p+q}$ holim $Y$. If the inverse system $Y$ lies in pro-Ho(Fin) or pro-Ho( $R$-Fin), then the $\lim p$-terms vanish for $p>0$, and

$$
\pi_{q} \text { holim } Y=\lim \text { pro- } \pi_{q} Y \text {. }
$$


Finiteness also implies that the induced isomorphism

$$
\lim \text { pro- } \pi_{q}\left({ }_{R} \circ X\right) \rightarrow \lim \text { pro- } \pi_{q}\left(\hat{R}_{R} \circ \text { holim } \circ{ }_{R} \circ X\right)
$$

induces an isomorphism before taking inverse limits. The Artin-Mazur Whitehead theorem (5) for pro-Ho(Top) now yields an equivalence on the Postnikov systems of

$$
\hat{R}_{R} \circ X \rightarrow{ }_{R}^{\wedge} \circ \text { holim } \circ{ }_{R} \circ X
$$

Recall (5) that the Postnikov system of an object $\left\{Y_{\alpha}\right\}$ of pro-Ho(Top) is the prospace $\left\{Y_{\alpha, s}\right\}$, where for each $\alpha,\left\{Y_{\alpha, s}\right\}$ is the Postnikov tower of $Y_{\alpha}$. Because objects of pro-Ho(Fin), and pro-Ho( $R$-Fin) are pro-isomorphic to their own Postnikov systems, the above map (3.8) is an isomorphism in pro-Ho(Top), as required.

(3.10) Proof of Theorem B, continued. The equivalence (3.8) has as its inverse the map

$$
\hat{R}_{R} \circ \text { holim } \circ{ }_{R}^{\wedge} \circ X \rightarrow{ }_{R}^{2} \circ X \quad\left(={ }_{R}^{\wedge} \circ X\right),
$$

obtained by applying $\hat{R}_{R}$ to the natural map

$$
\text { holim } \circ{ }_{R}^{\circ} \circ X \rightarrow{ }_{R} \circ X .
$$

The conclusion now follows from Theorem (3.3).

(3.12) REMARKS. Theorem B shows that the failure of all spaces to be $R$-good is a consequence of properties of holim. This failure may also be related to differences between the topologized and untopologized (ordinary) homotopy categories: The functor which forgets the Adams-Sullivan topologies may lose needed information. Two further examples and constructions illustrate this phenomenon. First, Bousfield's and Kan's examples of bad spaces [7, Chapter IV] arise from large fundamental groups and the failure of lim to commute with abelianization. Secondly, Bousfield [6] provides a different fix for $R$-bad spaces by iterating the Bousfield-Kan completion transfinitely to overcome these problems.

It is easy to see why nilpotent spaces are $R$-good [7, Chapter V] from this approach. If $X$ is nilpotent, then the Postnikov system of $X$ admits a refinement into towers of principal fibrations. Each fibre may be completed separately, and the resulting completions assembled. This can be done using the generalized prospaces described below.

4. Generalized prospaces. We define a category gpro-Top of generalized prospaces which will serve as a suitable target for an Artin-Mazur type completion and admit fibration and cofibration sequences. This category will be intermediate between Ho(pro-Top) which is too rigid to serve as a target for the Artin-Mazur completion and pro-Ho(Top) which is too loose to admit cofibres and fibres. Here are the main ideas.

(i) Allow as indexing categories small directed categories with product cones, but replace the requirement of equalizer cones by a requirement on the functors.

(ii) Develop the necessary properties of Artin-Mazur [5, Appendix] and Mardešić [see 12, Chapter 2] reindexing in this new setting. One more functor which replaces generalized prospaces by generalized prospaces with canonical homotopy equalizers will be required. 
(iii) Show that these reindexing techniques imply that any finite diagram without loops can be replaced by a similarly equivalent diagram of nice generalized prospaces, all indexed by the same cofinite small category, and levelwise maps.

(iv) Use the closed model structure of [12, Chapter 3] on categories of diagrams to define the required fibres, cofibres, etc. Some of this will be done in $\S 6$.

(4.1) Remarks. J. L. Aronson and D. A. Edwards [4] and A. Deleanu and P. Hilton [10] considered categories of generalized prospaces with no requirement on homotopy equalizers. Their constructions do not seem adequate for our purpose.

We shall define the category gpro-Top of generalized prospaces to be the category of (small) inverse systems over Top whose images as inverse systems over pro-Top lie in pro-Ho(Top). This will require particular care with Artin-Mazur and Mardešić reindexing techniques, which are discussed below.

We recall the definitions of product cones and homotopy equalizer cones. Given two spaces $X$ and $Y$, the diagram

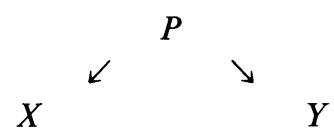

represents a product cone for $X$ and $Y$ if given any space $Z$ and maps $f: Z \rightarrow X$ and $g: Z \rightarrow Y$, there is an induced map $Z \rightarrow P$ such that $f$ and $g$ factor through $P$. Note: The induced map $Z \rightarrow P$ need not be unique; thus there are many distinct product cones.

Homotopy equalizer cones are defined analogously. Given maps $f, g: X \rightrightarrows Y$, a map $E \rightarrow Y$ is a homotopy equalizer cone for $f$ and $g$ if any map $h: W \rightarrow X$ with $f h \simeq g h$ factors through $E$ up to homotopy. Again, the factorization need not be unique; thus there are many distinct homotopy equalizer cones.

(4.2) Definition. A (small) inverse system $X$ over Top is a generalized prospace if $X$ admits

(i) product cones, and

(ii) homotopy equalizer cones.

(4.3) Definition. The category gpro-Top of generalized prospaces is the category whose objects are generalized prospaces and whose morphisms are defined by the Artin-Mazur [5, Appendix] formula

$$
\operatorname{gpro-Top}\left(\left\{X_{i}\right\},\left\{Y_{j}\right\}\right)=\lim _{j} \underset{i}{\operatorname{colim}} \operatorname{Top}\left(X_{i}, Y_{j}\right) \text {. }
$$

We weaken the Artin-Mazur [5, Appendix] definition of cofinal functor in analogy with Definition (4.2).

(4.4) Definition. Let $X$ be a generalized prospace, and $T: I \rightarrow J$ a functor taking values in the indexing category $J$ of $X$. We call $T$ cofinal if for every object $j$ of $J$, there is an object $i$ of $I$ and a morphism $T i \rightarrow j$ in $J$, and if for every pair of maps $T i \rightrightarrows j$ in $J$ there is a map $i^{\prime} \rightarrow i$ in $I$ such that the map $X\left(T i^{\prime}\right) \rightarrow X(T i)$ is a homotopy equalizer cone of the maps $X(T i) \rightrightarrows X(j)$.

(4.5) Proposition. The functor $\pi$ : pro-Top $\rightarrow$ pro-Ho(Top) factors naturally through gpro-Top. 
The proof is easy and omitted.

(4.6) REMARKS. (i) Let $X$ be a generalized prospace, regarded as a functor from an indexing category $I$ to Top. Note that the existence of product cones is a condition that $I$ must satisfy, whereas the existence of homotopy equalizer cones is a restriction on $X$. Proposition (4.4) implies that a suitable quotient category $I / \equiv$ admits equalizer cones, and fits into a commutative diagrm of categories and functors

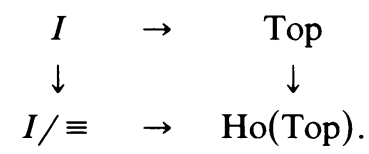

(ii) Cofinal functors need not induce isomorphisms of generalized prospaces, unlike the usual situation for procategories. However, the homotopy equalizer conditions easily imply the following.

(4.7) Proposition. Let $X$ be a generalized prospace, and $T: I \rightarrow J$ be a cofinal functor from $I$ to the indexing category $J$ of $X$. Then $X \circ T$ is a generalized prospace indexed by $I, T$ induces a cofinal functor in the Artin-Mazur sense on suitable quotients of $I$ and $J$, and the map $X \rightarrow X \circ T$ becomes an isomorphism in pro-Ho(Top).

We shall now define the homotopy category Ho(gpro-Top). Recall the definition of the prohomotopy progroups.

$$
\text { pro- } \pi_{n}\left(\left\{X_{i}\right\}\right)=\left\{\pi_{n}\left(X_{i}\right)\right\} \text {. }
$$

(4.9) Definition. A map in gpro-Top is a pro- $\pi_{*}$-equivalence if it induces isomorphisms on prohomotopy groups. The homotopy category of generalized prospaces Ho(gpro-Top) is the localization of gpro-Top at the class of pro- $\pi_{*}$-equivalences.

(4.10) Remarks. Let $f: X \rightarrow Y$ be a map of prospaces which is a pro- $\pi_{*}$-equivalence. Edwards and I [12, Chapter 5] have shown that $f$ need not be a homotopy equivalence, and need not even induce a homotopy equivalence in pro-Ho(Top). Also, it is still unknown whether the canonical projection from $\mathrm{Ho}$ (pro-Top) to pro-Ho(Top) reflects isomorphisms. However, both this property and the Whitehead theorem hold for suitably restricted objects, including profinite spaces and Postnikov systems. Therefore Definition (4.9) suffices for our purposes. In addition, it is easy to prove the following (the second part is due to Artin and Mazur [5]; the proof of the first part is similar and omitted).

(4.11) Proposition. Let $f: X \rightarrow Y$ be a pro- $\pi_{*}$-equivalence in pro-Ho(Top). Then the extended Artin-Mazur completion of $\$ 2$ induces an equivalence in pro-Ho(Top) $f_{\mathrm{AM}}^{\wedge}: X \rightarrow Y$, and similarly for the p-completion ${ }_{p}$, the $R$-completion ${ }_{R}$, and the Postnikov system functor in pro-Ho(Top).

Therefore, pro- $\pi_{*}$-equivalences in gpro-Top, which induce pro- $\pi_{*}$-equivalences in pro-Ho(Top), also induce natural equivalences in the sense of Artin and Mazur.

(4.12) Proposition. The functor $\pi$ : Ho(pro-Top) $\rightarrow$ pro-Ho(Top) (pro $=\pi_{*-}$ equivalences) factor naturally through $\mathrm{Ho}(\mathrm{gpro}-\mathrm{Top})$. 
The proof is easy and omitted.

We now review Artin-Mazur [5, Appendix] reindexing and adapt Mardešić reindexing to the case of gpro-Top. Artin-Mazur reindexing is used to replace finite diagrams without loops in gpro-Top by finite diagrams with level preserving maps. The usual construction as applied to pro-Top also works in gpro-Top because we only require that the "domain" and "range" functors induce cofinal functors in the sense of Definition (4.4).

Usually, the Mardešić construction is applied to a prospace in order to obtain a prospace indexed by a cofinite, strongly directed set. However, the Mardešić construction may be applied to any inverse system of spaces. We shall use the following version of the Mardešić construction.

(4.13) Definition (COMPARE [12, Chapter 2]). Let $X$ be a generalized prospace with indexing category I. Applying the Mardešić construction $M$ to $X$ yields an inverse system $M X$ with the following indexing category $M I$. Objects of $M I$ are finite diagrams in I with distinguished "homotopy initial objects", denoted init ( ), that is, initial objects up to homotopy for the images under $X$. If $D$ and $D^{\prime}$ are such finite diagrams in $I$, and $D^{\prime}$ is a subdiagram of $D$, then there is one morphism in $M I$ from $D$ to $D^{\prime}$ for each composite map from the homotopy $\operatorname{init}(D)$ to $\operatorname{init}\left(D^{\prime}\right)$. The inverse system $M X$ associates to each diagram $D$ the object $Y(\operatorname{init}(D))$, and to each morphism $D \rightarrow D^{\prime}$ the corresponding morphism init $(D) \rightarrow \operatorname{init}\left(D^{\prime}\right)$.

(4.14) Proposition. The Mardešić construction induces functors

$$
\begin{aligned}
& M: \text { Ho(gpro-Top) } \rightarrow \text { Ho(gpro-Top), } \\
& M: \text { gpro-Top } \rightarrow \text { gpro-Top, }
\end{aligned}
$$

and a natural transformation $M \rightarrow \mathrm{id}$.

Proof. The proof is an easy modification of the analogous results for the Edwards-Hastings [12, Chapter 2] version of the usual Mardešic construction. We therefore leave most of the details to the reader. Functoriality on gpro-Top follows directly from the definition, as does existence of the natural transformation $M \rightarrow$ id.

It is easy to check that $M$ preserves pro- $\pi_{*}$-equivalences, and thus induces a functor on Ho(gpro-Top).

It is easy to see that each object of $M X$ has only finitely many predecessors. We call the indexing category of such an object cofinite. In addition, if $m \rightarrow m^{\prime} \rightarrow m$ are morphisms in the indexing category of $M I$, then $m=m^{\prime}$, and both morphisms are the identity. We call a cofinite indexing category with this additional property inductive. All of the arguments of [12, Chapter 3] which used induction on cofinite, strongly directed indexing categories may be performed over these inductive categories provided that care is taken with fibrations and fibrant objects.

We now consider the effect of applying first Artin-Mazur reindexing and then Mardešić reindexing to a finite diagram without loops. This process yields an equivalent (over Ho(gpro-Top)) finite diagram in which each object is indexed by the same cofinite category and all maps preserve levels.

We shall need canonical homotopy equalizer cones to construct cofibres and fibres in gpro-Top. 
(4.15) Notation. Let $f, g: X \rightrightarrows Y$ be maps in Top. The standard homotopy equalizer of $f$ and $g$ is the map $P \rightarrow X$ defined by the pullback diagram

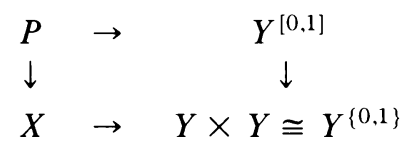

(4.16) Definition. We shall call a generalized prospace $X$ nice if, for any pair of bonding maps $X_{i} \rightrightarrows X_{j}$, their standard homotopy equalizer is also a bonding map of $X$.

It is easy to show that any generalized prospace $X$ may be replaced canonically by a nice space by first applying the above Mardešić construction and then adjoining standard homotopy equalizers.

(4.17) Construction. For each cofinite inverse system $X$, define a nice inverse system $N X$ as follows. By induction on the number of successors, for each pair of bonding maps $X_{i} \rightrightarrows X_{j}$, adjoin their standard homotopy equalizer $P$ and bonding map $P \rightarrow X_{i}$. Also, adjoin a bonding map $X_{k} \rightarrow P$ factoring at least one homotopy equalizer cone $X_{k} \rightarrow X_{i}$ for the original bonding maps. Continue inductively to obtain $N X$.

(4.18) Proposition. $X$ and $N X$ are mutually cofinal.

The proof is easy and omitted.

(4.19) Proposition. For any finite diagram without loops over gpro-Top, we may construct an equivalent diagram in which

(i) all objects are indexed by the same cofinite indexing category,

(ii) all maps preserve levels, and,

(iii) all objects are nice.

Further, any two such diagrams are naturally equivalent.

Proof. Apply successively the above constructions for first Artin-Mazur reindexing, then Mardešić reindexing, and finally for replacing each object by a nice object, taking predecessors of originally chosen homotopy equalizer cones where necessary. For the final claim, it is only necessary to note that any two choices of homotopy equalizer cones have a common predecessor in each object, and then a common predecessor level in the indexing category. The objects associated to this index are also the required homotopy equalizer cones.

In $§ 6$ we shall define fibres and cofibres in gpro-Top. By applying as much of the above reindexing as necessary, we need only define the fibre and cofibre of levelwise maps, and then verify that the constructions are well defined up to natural equivalence in $\mathrm{Ho}$ (gpro-Top).

5. The Artin-Mazur completion on generalized prospaces. We first lift the ArtinMazur $R$-completion (on pro-Ho(Top)) to a rigid Artin-Mazur completion $\hat{r i g}_{\text {: }}$ : gpro-Top $\rightarrow$ gpro-Top, inducing a similar completion on Ho(gpro-Top). We then observe that these rigid completions are idempotent. Both Theorems $\mathrm{A}$ and $\mathrm{B}$ hold in this rigid setting. We shall study the behavior of the rigid Artin-Mazur $R$-completion 
on cofibrations and fibrations in this rigid setting in later sections. Similar results hold for the finite and $p$-completions; we shall not state these explicitly.

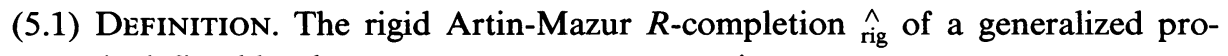
space $X$ is defined by the comma category construction

$$
X_{\text {rig }}^{\wedge}=\{X \downarrow R \text {-Fin } \rightarrow R \text {-Fin }\} .
$$

(5.2) LeMMA. $\{X \downarrow R$-Fin $\rightarrow R$-Fin $\}$ lies in gpro-Top.

Proof. As in the proof of the corresponding assertion for the weak prohomotopy category, Lemma (2.5), the indexing category $X \downarrow R$-Fin admits product cones and homotopy equalizer cones. The conclusion follows.

As in $\S 2$, the construction ${ }_{\text {rig }}$ extends to a functor $\underset{\text { rig }}{\wedge}$ gpro-Top $\rightarrow$ gpro-Top, and induces a functor, also denoted $\underset{\text { rig }}{\wedge}$, from $\mathrm{Ho}$ (gpro-Top) to itself. It is now easy to see that:

(i) Both functors $\underset{\text { rig }}{\wedge}$ are idempotent-this follows from the comma category definition.

(ii) The above functors map onto the appropriate full subcategories generated by pro-objects over $R$-Fin, and are adjoint to the inclusions

$$
j: \text { gpro- } R \text {-Fin } \rightarrow \text { gpro-Top and } j: \text { Ho(gpro- } R \text {-Fin) } \rightarrow \text { Ho(gpro-Top). }
$$

We shall use the following proposition about homotopy limits to prove a rigid version of Theorem $\mathrm{B}$.

(5.3) Proposition. There is a homotopy limit holim: Ho(gpro-R-Fin) $\rightarrow$ Ho(Top) which satisfies the standard adjointness relation

$$
\operatorname{Ho}(\operatorname{Top})\left(X, \operatorname{holim}\left\{F_{i}\right\}\right) \cong \operatorname{Ho}(\text { gpro-Top })\left(J X,\left\{F_{i}\right\}\right),
$$

where $X \in \mathrm{Top},\left\{F_{i}\right\} \in$ gpro-Top, and $J: \mathrm{Ho}(\mathrm{Top}) \rightarrow$ Ho(gpro-Top) is the inclusion.

Proof (Compare (3.5) AND the existence of Sullivan limits [23, 24]). We may define holim for any cofinite category of diagrams using [12, Chapter 4]. We then use the Bousfield-Kan spectral sequence and the vanishing of $\lim ^{p}$ pro- $\pi_{n}\left\{F_{i}\right\}$, $p>0$, for $\left\{F_{i}\right\} \in$ gpro- $R$-Fin to show that these constructions induce the required functor on $\mathrm{Ho}$ (gpro- $R$-Fin). Adjointness follows from adjointness of holim for categories of diagrams; see [12, Chapter 4].

Therefore the Sullivan completion factors as

$$
\text { Ho(Top) } \stackrel{J}{\rightarrow} \text { Ho (gpro-Top) } \stackrel{\hat{\text { rig }} \rightarrow}{\rightarrow} \text { Ho(gpro-R-Fin) } \stackrel{\text { holim }}{\rightarrow} \text { Ho(Top), }
$$

and $\underset{\text { rig }}{\wedge}$ is a reflection.

This yields a factorization of the Sullivan completion through an idempotent completion.

6. Fibration and cofibration sequences. We outline a proposed theory of cofibration and fibration sequences in gpro-Top, and prove that the $R$-completions of cofibres and fibres are well defined. Although we do not need the more general result that cofibres and fibres are well defined for this paper, we conjecture that it 
holds and we supply a heuristic sketch of a proof. Recall the techniques used in [12, Chapter 3] to define fibrations and cofibrations in pro-Top. We proceed as follows.

(i) Define fibrations and cofibrations for suitable categories of diagrams. The constructions in [12, Chapter 3] appear to require that the diagrams be indexed by cofinite, strongly directed sets, but these requirements can actually be reduced somewhat to handle our case. We need only require that the indexing categories be cofinite, and that given any pair of maps $j \rightarrow k \rightarrow j$ in an indexing category, that $j=k$ and both maps are isomorphisms. Recall that these indexing categories are called inductive.

(ii) Use Artin-Mazur and Mardešić reindexing to replace any promap by a levelwise map of prospaces indexed by cofinite, strongly directed sets. We have shown, in $\S 4$, how to apply these reindexing techniques to gpro-Top. In this case we replace any promap by a levelwise map of prospaces indexed by cofinite, inductive categories.

(iii) Define the fibre and cofibre of a promap to be the fibre and cofibre of the levelwise maps obtained in (ii) above. One must then prove that the fibre and cofibre are again prospaces and are well defined up to equivalence in gpro-Top. The first condition is trivial in the case where the indexing categories are cofinite, strongly directed sets, and is only slightly more difficult in the present case.

Similarly, the second condition is relatively straightforward in the case of prospaces. For generalized prospaces, it is easy to see that fibres are well defined up to pro- $\pi_{*}$-equivalence, and cofibres are well defined up to cohomology equivalence.

(6.1) Proposition. Let $D$ be an inductive indexing category. Then the functor category Top $^{D}$ is a closed model category. Also the fibres and cofibres of maps in Top ${ }^{D}$ of objects which admit the homotopy equalizer cones required for generalized prospaces themselves admit these homotopy equalizer cones.

Proof. For the first assertion, follow the proof in [12, Chapter 3] that Top $^{D}$ is a closed model category if $D$ is a cofinite, strongly directed set. We omit the details because we shall not need this generality below.

For the second assertion, first consider a diagram in Top in which each horizontal map is a fibration, and $E^{\prime}$ and $B^{\prime}$ are canonical homotopy equalizers. Then there is a map $E^{\prime} \rightarrow B^{\prime}$, induced from the construction of canonical homotopy equalizers, which is a fibration, whose fibre is again a (canonical) homotopy equalizer. If the horizontal maps are assumed to be cofibrations, then a similar result holds except that we only obtain a homotopy equalizer cone. (These are easy versions of glueing and coglueing theorems of R. Brown and P. E. Heath [8]; see also [14].) The second assertion now follows from the niceness construction of $\S 4$.

(6.2) Proposition. The fibre of a fibration in gpro-Top is well defined up to pro- $\pi_{*}$-equivalence.

Proof. All of the constructions involved in replacing a map of prospaces by a levelwise map of nice generalized prospaces, indexed by an inductive indexing category, use maps which become equivalences in pro-Ho(Top). Therefore, these 
maps are pro- $\pi_{*}$-equivalences. The conclusion now follows from the five-lemma and exactness of the pro- $\pi_{*}$-sequence of a fibration.

(6.3) Proposition. The cofibre of a map of generalized prospaces is well defined up to cohomology equivalence.

Proof. Follow the proof of Proposition (6.2), except for replacing pro- $\pi_{*}$ equivalences by cohomology equivalences, and replacing fibrations by cofibrations. The conclusion follows.

(6.4) Corollary to Propositions (6.2) AND (6.3). The R-completions of fibres and cofibres are well defined up to pro- $\pi_{*}$-equivalence, similarly for the finite and $p$ completions.

(6.5) REMARKs. We conjecture that fibres and cofibres are defined up to isomorphism in Ho(gpro-Top), that is, up to pro-Ho(Top)-isomorphism. Compare the results of [12, Chapter 3] for pro-Top. Because the Whitehead theorem fails in general for pro-Ho(Top) [12, Chapter 5], this conjecture does not follow from Propositions (6.2) and (6.3) above. Instead careful glueing and coglueing arguments appear to be required. See [8], and also some proofs of a restricted Whitehead theorem for pro-Ho(Top) [5], also M. Mosynska [19], Mardešić [18], and D. A. Edwards and R. Geoghegan [11].

7. Proofs of Theorems C and D. We shall prove that the rigid S. Artin-Mazur completion, regarded as a functor into gpro- $R$-Fin, preserves both fibration and cofibration sequences. The fibre in gpro- $R$-Fin of a map in gpro- $R$-Fin is defined as in gpro-Top. However, the cofibre in gpro-Top of a map in gpro- $R$-Fin need not lie in gpro- $R$-Fin. This difficulty is easily rectified by applying the rigid completion $\hat{\text { rig }}$ again since it is a reflection. Again, similar results hold for the finite and p-completions.

(7.1) THEOREM C. The rigid Artin-Mazur completion preserves fibrations.

Proof. Let $F \rightarrow E \rightarrow B$ be an inverse system of fibrations over Top, which is a fibration in gpro-Top. Consider all fibrations of $R$-finite spaces $F_{i} \rightarrow E_{i} \rightarrow B_{i}$ under $F \rightarrow E \rightarrow B$. These form a category, together with functors to the objects $\left\{F_{i}\right\}$, $\left\{E_{i}\right\}$, and $\left\{B_{i}\right\}$, which lie in gpro-Top (easy check). There are also natural maps $F_{\text {rig }}^{\wedge} \rightarrow\left\{F_{i}\right\}, E_{\text {rig }}^{\wedge} \rightarrow\left\{E_{i}\right\}$, and $B_{\text {rig }}^{\wedge} \rightarrow\left\{B_{i}\right\}$. The last two maps are easily seen to be cofinal, hence pro- $\pi_{*}$-equivalences. The first map is then also a pro- $\pi_{*}$-equivalence by the five-lemma because the second two maps induce isomorphisms on prohomotopy. The conclusion follows.

\section{(7.2) TheOREM D. The rigid Artin-Mazur completion preserves cofibrations.}

Proof. In essence, follow the proof of Theorem $C$, except that the fibre sequences of $R$-finite spaces are replaced by approximations to cofibre sequences of $R$-finite spaces. Let $A \rightarrow X \rightarrow C$ be an inverse system of cofibre sequences in Top which is a cofibration in gpro-Top. Consider the category of all sequences $A_{i} \rightarrow X_{i} \rightarrow C_{i}$ where the map $A \rightarrow X$ is a cofibration of $R$-finite spaces, and $C$ is an $R$-finite space under 
the cofibre $X / A(X / A$ is not in general an $R$-finite space). As in the proof of Theorem $\mathrm{C}$, this yields inverse systems of $R$-finite spaces which lie in gpro-Top, and are cofinal in the rigid Artin-Mazur $R$-completions of $A, X$, and $C$, respectively. Finally, identify $C$ as the rigid Artin-Mazur completion of the cofibre of the map $A \rightarrow A$. The conclusion follows.

(7.3) Remarks. Recall that the Sullivan completion is the homotopy limit of the rigid Artin-Mazur completion, and that homotopy limits preserve fibrations but need not preserve cofibrations (cf. [12, Chapter 4] for the homotopy limits). Thus Theorem C implies Sullivan's [24] result that the Sullivan completion preserves fibrations, and Theorem D and Sullivan's example that $\hat{\text { Sul }}$ does not preserve cofibrations imply that the problem lies in the homotopy limits.

\section{REFERENCES}

1. J. F. Adams, Adams's problems, Manifolds-Tokyo 1973, Univ. of Tokyo Press, Tokyo, 1975, pp. $430-431$.

2. Idempotent functors in homotopy theory, Manifolds-Tokyo 1973, Univ. of Tokyo Press, Tokyo, 1975, pp. 247-253.

3. Localization and completion, Lecture Notes in Math., Univ. of Chicago, 1975.

4. J. L. Aronson and D. A. Edwards, The mathematical foundations of quantum mechanics, Synthèse 42 (1979), 1-70.

5. M. Artin and B. Mazur, Étale homotopy theory, Lecture Notes in Math., Vol. 100, Springer, New York, 1969.

6. A. K. Bousfield, The localization of spaces with respect to homology, Topology 14 (1975), 133-150.

7. A. K. Bousfield and D. M. Kan, Homotopy limits, completions, and localizations, Lecture Notes in Math., Vol. 304, Springer, New York, 1973.

8. R. Brown and P. R. Heath, Coglueing homotopy equivalences, Math. Z. 113 (1970), 313-325.

9. A. Deleanu, Topologized objects in categories and the Sullivan profinite completion, J. Pure Appl. Algebra 25 (1982), 21-24.

10. A. Deleanu and P. Hilton, Generalized shape theory, General Topology and Its Relations to Modern Analysis and Algebra. IV (J. Novak, ed.), Lecture Notes in Math., Vol. 609, Springer, New York, 1977. pp. $56-65$.

11. D. A. Edwards and R. Geoghegan, The stability problem in shape and a Whitehead theorem in prohomotopy, Trans. Amer. Math. Soc. 214 (1975), 261-277.

12. D. A. Edwards and H. M. Hastings, Čech and Steenrod homotopy theory with applications to geometric topology, Lecture Notes in Math., Vol. 542, Springer, New York, 1976.

13. W_ Why the R-completion works, General Topology Appl. 7 (1977), 179-184.

14. H. M. Hastings, Fibrations of compactly generated spaces, Michigan Math. J. 21 (1974), 243-251.

15. Steenrod homotopy theory, homotopy idempotents, and homotopy limits, Topology Proc. 2 (1977), $461-477$.

16. Ju. T. Lisica and S. Mardešić, Coherent prohomotopy and strong shape, Zagreb, 1982, preprint.

17. S. Mac Lane, Categories for the working mathematician, Graduate Texts in Math., vol. 5, Springer, New York, 1971.

18. S. Mardešić, On the Whitehead theorem in shape theory. I, II, Fund. Math. 91 (1976), 51-64, 93-103.

19. M. Mosynska, The Whitehead theorem in the theory of shapes, Fund. Math. 80 (1973), 235-240.

20. T. Porter, Coherent prohomotopy theory, Cahiers Topologie Géom. Différentielle 19 (1978), 3-46.

21. D. Quillen, Homotopical algebra, Lecture Notes in Math., Vol. 43, Springer, New York, 1967.

22. __ An application of simplicial profinite groups, Comment. Math. Helv. 44 (1969), 45-60.

23. D. Sullivan, Geometric topology. I: Localization, periodicity, and Galois symmetry, Notes, M.I.T., 1971.

24. Genetics of homotopy theory and the Adams conjecture, Ann. of Math. (2) 100 (1974), 1-80.

25. R. Vogt, Homotopy limits and colimits, Math. Z. 134 (1973), 11-52.

Department of Mathematics, Hofstra University, Hempstead, New York 11550 